\title{
Óleos essenciais da Amazônia VII. (1)
}

Otto R. Gottlieb $\left({ }^{2}\right)$; Midori Koketsu $\left({ }^{3}\right)$; Mauro T. Magalhães $\left({ }^{3}\right)$; J. Guilherme S. Maia $\left({ }^{4}\right)$; Paulo H. Mendes $\left({ }^{3}\right) ;$ A. I. da Rocha $\left({ }^{5}\right)$; Miriam L. da Silva (4); Viktor C. Wiiberg $\left({ }^{3}\right)$

\section{Resumo}

São descritos, neste trabalho, os principais constituintes dos óleos essenciais de algumas espécies amazônicas pertencentes às famílias Annonaceae, Bignoniaceae, Burseraceae, Compositae, Euphorbiaceae, Labiatae, Lauraceae, Leguminosae-Papilionoideae, Myrtaceae, Piperaceae e Zingiberaceae.

\section{INTRODUÇÃO}

Em continuação ao projeto que visa à triagem da flora odorífera da Amazônia com o objetivo de encontrar novas fontes de óleos essenciais comercializáveis (Alencar et al., 1971; Araújo et al., 1971; Morais et al., 1972 a, b; Corrêa et al., 1972; Silva et al., 1973), o presente trabalho apresenta monografias referentes a 25 espécies pertencentes a 11 famílias (Tabelas).

\section{BIGNONIACEAE}

Tanaecium nocturnum, o "carimbó da meta", é uma trepadeira. Todas as suas partes cheiram a amendoas amargas. O presente es tudo mostra que o óleo essencial do caule é, de fato, constituído quase exclusivamente de aldeido benzóico. As flores, que desabrocham ao anoitecer e caem ao raiar do dia, são aproveitadas para aromatizar roupa. A casca passa por ser útil em distúrbios gástricos.

\section{COIMPOSITAE}

Ichthyothere cunabi, o "cunabi", uma erva perene de cheiro penetrante, cultivada pelos indígenas, é usada em pesca. As estruturas dos constituintes ictiotóxicos são conhecidas (Mors et al., 1965).

\section{LAURACEAE}

As aproximadamente 40 espécies pertencentes ao gênero Aniba podem ser divididas de acordo com a natureza química do constituinte predominante do respectivo óleo essencial, em três grupos: o grupo de linalol ( $A$. rosaedora Ducke, A. duckei Kosterm.); o grupo do benzoato, (A. fragrans Ducke, A. firmula (Nees et Mart.) Mez, A. gardneri (Meissn.) Mez, A. burchellii Kosterm., A. parviflora (Meissn.) Mez, A. permollis (Nees) Mez, A. guianensis Aubl.) e o grupo de alilbenzeno ( $A$. canelilla (H.B.K.) Mez, A. hostmanniana (Ness) Mez, A. pseudocoto (Reesby) Kosterm. (Moraes et al., 1972 a). Dada a conhecida dificulda. de de classificação de espécies do gênero, é possível que várias das amostras utilizadas nas análises não tenham sido determinadas corretamente. A classificação química em três grupos, no entanto, encontra apoio nos novos dados da Tabela. A. burchellii, para a qual o trabalho anterior assinala apenas o isolamento de benzoato de benzila, por cromatografia de um extrato da madeira em coluna de sílica, mostra possuir alto teor em alilbenzenos e até um propenilbenzeno (Alvarenga et al., 1977). Este fato não é surpreendente, já que as substâncias particulares detectadas coocorrem com diversos oxidativos de alil-e propenilbenzenos, ou seja neolignanas, abundantemente representadâs na espécie (Alvarenga et al., 1977).

(1) - Este trabalho é amparado pelo BASA, CNPq e EMBRAPA.

(2) - Instituto de Química, USP, SP.

( ${ }^{3}$ ) - Centro de Tecnologia Agrícola e Alimentar, EMBRAPA, RJ.

(4) - Instituto Nacional de Pesquisas da Amazônia, Manaus.

(5) - Departamento de Química, ICE/FUA - AM. 
A análise de dois óleos essenciais de $A$. parviflora (Tabela), também mostra que composição pode variar de acordo com o órgão da planta.

Benzoato de benzila foi anteriormente localizado em Cinnamomum zeylanicum Blume (Gottlieb, 1972) e Ocotea teleiandra (Meissn.) Mez (Naves et al., 1961). Desta forma, sua presença em Ocotea não é estranhável. Por ora, no entanto, não cabe confiança no dado da Tabela com respeito a Ocotea sp. $35 / 73$ já que este gênero é de difícil determinação em Lauraceae.

A casca de uma espécie de Nectandra não classificada contém safrol, o que, de novo, não é surpreendente, pois os componentes fixos mais importantes do seu lenho são neolignanas (Braz Filho et al., 1980).

\section{LEGUMTNOSAE-PAPILIONOIDEAE}

Um decocto da amêndoa da semente do Monopteryx uaucu é utilizado em medicina popular. Mesmo que esta amêndoa não tenha sido analisada no decurso do presente traba-
Iho, fornece a composição do óleo essencial da madeira do tronco (Tabela) um indício da sua composição química. Os constituintes predominantes no óleo são alibenzenos que possuem reconhecida atividade biológica.

\section{PIPERACEA}

O óleo essencial de Piper marginatum Jacq. uma espécie analisada anteriormente, contém ac lado do safrol $(4,2 \%)$ etilpiperonilcetona $(25,0 \%)$ (Silva et al., 1973) e outras propiofenonás (Diaz \& Gottlieb, 1979).

No trabalho Alencar et al., 1971, consideramos possivel a exploração do "panquilé", como fonte de produção de safrol. A classificação da espécie como Piper cavalcantei Yuncker, no entanto, precisa ser revista. A análise do Panquilé é repetida na tabela acrescido da classificação correta, Piper callosum.

Todos os outros dados sobre Piperaceae constantes da Tabela, inclusive Piper aduncum var. aduncum, o "piperão", e $P$. hispidinervium, a "pimenta longa", são inéditos.

TABELA - Composição percentual e características de alguns óleos essenciais da Amazônia.

\section{FAMILIA}

Espécie

Parte Vegetal

N.० de Herbário *

Local de Coleta
Composiçāo percentual

do Oleo Essencial
Rend. \%

\section{ANNONACEAE}

Xylopia polyantha - R. E. Fries

Folhas da árvore

IAN

Belém, PA

$\begin{array}{lr}\text { Cinamaldeido } & 7,9 \\ \propto \text { - Pineno } & 2,8 \\ \beta \text { - Pineno } & 2,2 \\ \text { Monoterpenos } & 60,9 \\ \text { Sesquiterpenos } & 20,2\end{array}$

Benzaldeido

Monoterpenos
0,10

1,5048

2,2

20,2

\section{6,0}

0,28

1,5497

1,0534

3,0

Bur. et K. Sh.

Caule do cipó

IAN 128049

Belém, PA

Gottlieb et al. 
(Continuação TABELA 1)

\section{BURSERACEAE}

Protium paraense Cuatr.

Casca da árvore

IAN 125677

!garapé Aurá, Belém, PA

\section{COMPOSITAE}

Ichthyothere cunabi Mart.

Folhas da Erva

INPA cf. 2440

Manaus, AM

\section{EUPHORBIACEAE}

Craton matourensis Aubl.

Casca da erva

IAN 63387

Igarapé Amazonas, Belém, PA

\section{LABIATAE}

Hyptis goyazensis Benth.

Folhas e galhos do subarbusto

IAN 112953; P. 13280

Rio Madeira, AM

Hyptis suaveolens Poit.

Folhas da erva

INPA (material botânico destruído em incêndio)

Manaus, AM

\section{LAURACEAE}

Aniba burchellii Kosterm.

Folhas da árvore

P. 13274

Humaitá, AM

Aniba $\mathrm{sp.}$

Madeira da árvore

P. 13302

jenaro Herrera, Peru

Aniba parviflora (Meissn) Mez

Folhas da árvore

IAN 11620

Museu Goeldi, Belém, PA

Aniba parviflora (Meissn.) Mez

Raiz da árvore

IAN 11620

Museu Goeldi, Belém, PA $\propto$ - Pineno $\quad 50,5$

$\beta$ - Pineno

Felandireno

p-Cimeno

Monoterpenos

Sesquiterpenos

0,16

1,4830

0,8990

Felandireno

0,8

Limoneno

$\propto$ - Pineno

Monoterpenos

Sesquiterpenos

1,4929

0,9936

$\propto$-Pineno

Elemicina

p - Cimeno

Felandreno

Monoterpenos

Sesquiterpenos

1,8-Cineol

0,47

$\propto$ - Pineno

Cânfora

1,4719

$\beta$ - Pineno

0,9121

Moncterpenos

0,43

1,8-Cineol

$\alpha$-Pineno

$\beta$ - Pineno

Felandreno

1,4831

Sesquiterpenos

0.8879

Salicilato de benzila

0.15

$\propto$ - Pineno

1,5254

Benzoato de benzila

0,9399

Monoterpenos

Sesquiterpenos

0,93

Safiol

Isossafrol

Metileugenol

Eugenol

Monoterpenos

Salicilato de benzila

Benzuato de benzila

0,9942

- Pineno

Monoterpenos

Sesquiterpenos

Benzoato de benzila Benzaldeido

oleos. 
Licaria sp $63 / 74$

Madeira da árvore

INPA 43576

Reserva Ducke, Manaus, AM

Nectandra sp.

Casca da árvore

INPA 46534

Rosa de Maio, Km 8 da Estr. AM-10

(Manaus - Itacoatiara), AM.

Ocotea petalanthera (Meissn.) Mez

Folha da árvore

IAN

Acará e Moju, PA

Ocotea sp $35 / 73$

Madeira da árvore

INPA 42240 e 14524

Manaus, AM

\section{LEGUMINOSAE-PAPILIONOIDEAE}

Monopteryx uaucu Spruce

Madeira da árvore

IAN 146516

Uaupés, AM

Ormosia flava (Ducke) Rudd.

Niadeira da árvore

IAN 150706

Belém, PA

\section{MYRTACEAE}

Myrcia cuprea Kiaers.

Folhas do arbusto

IAN 140412

Belém, PA

\section{PIPERACEAE}

Piper aduncum L. var. aduncum Folhas do arbusto

IAN 66735

Benfica, PA

Piper aduncum var. cordulatum

(C. DC.) Yuncker

Folhas do arbusto

INPA 43458

Manaus, AM

Piper callosum R. et Pav.

Folhas do arbusto

INPA 88603

Manaus, Km 7 da Estr. AM-10

(Manaus-Itacoatiara), AM. $\alpha$ - Pineno $\quad 6,8$

$\beta$ - Pineno $\quad 4,9$

0,12

Linalol

1,5044

Nerolidol

0,9912

Monoterpenos

Sesquiterpenos

0,37

$\propto$ - Pineno

$\beta$ - Pineno

1,4992

Safrol

0,9613

Sesquiterpenos

0,94

$\beta$ - Pineno

$\mathrm{p}$ - Cimeno

$\propto$ - Pineno

1,4850

Monoterpenos

Sesquiterpenos

0,9309

Benzoato de benzila

Salicilato de benzila

Sesquiterpenos

43,9

82,0

0,34

traços $\quad 1,5630$

$17,9 \quad 1,1600$

Anetol

Metilchavicol

Metileugenol

13,7

2,0

1,5061

Elemicina

0,9756

Monoterpenos

Sesquiterpenos

Benzaldeido

Benzoato de benzila

1,48

Salicilato de benzila

1,5767

1,1808

Álcool sesquiterpênico

67,8

Nerolidol

Monoterpenos

Sesquiterpenos

0,04

4,8

16,6

10,7

Dilapiol

74,5

1,43

Cânfora

traços

1,5170

Monoterpenos

Sesquiterpenos

14,1

1,0976

11,4

Dilapiol

88,4

Monoterpenos

Sesquiterpenos

5,0

3,5

6,6

, 5240

1,1318

Safrol

69,0

1,9

Metileugenol

1,5100

Terpenos
8,0

23,0 
Folhas do arbusto

INPA 43457; 39754

Manaus, AM

Piper ottonoides Yuncker

Folhas do arbusto

IAN
Piper hispidinervium C. DC.

Moju-Acará, PA

$\begin{array}{lrl}\text { Safrol } & 89,0 & 3,0 \\ \text { Monoterpenos } & 11,0 & 1,5325 \\ & & 1,0843 \\ & & \\ \text { Cariofileno } & 6,3 & 0,50 \\ \beta \text { - Pineno } & 4,6 & 1,5000 \\ \propto \text { - Terpineno } & 4,4 & 0,9633 \\ \text { p-Cirreno } & 1,8 & \\ \propto \text { - Pineno } & 1,0 & \\ \text { Monoterpenos } & 82,6 & \end{array}$

ZINGIBERACEAE

Alpinia speciosa (Wendl.) Schum.

Folhas e galhos

INPA 48611

Cambixe - AM

$\begin{array}{lrl}\propto \text { - Pineno } & 6,5 & 0,35 \\ \beta \text {-Pineno } & 5,9 & 1,4802 \\ 1,8 \text { - Cineol } & 22,4 & 0,9407 \\ \text { p-Cimeno } & 20,8 & \\ \text { Sesquiterpenos } & 44,3 & \end{array}$

$\left({ }^{\circ}\right)$ - Herbários: INPA, CNPq, Manaus; IAN, Centro de Pesquisa Agropecuária do Trópico Úmido, EMBRAPA, Belém; $\mathrm{Pa}$., Coleção particular do Dr. J. M. Pires, Belém.

\section{PARTE EXPERIMENTAL}

Obtenção dos óleos essenciais: Os óleos essenciais foram obtidos por arraste a vapor com coobação das águas condensadas, em aparelho de Clevenger modificado (Gottlieb \& Magalhães, 1960)

Separação e obtenção de constituintes de óleos essenciais: A separação e obtenção de componentes de óleos essenciais foi feita por cromatografia gás-líquido preparativa utilizando-se um Cromatógrafo Varian modelo $2440 \mathrm{~S}$, operando com coluna de $3 / 8 " 8 \times 6 \mathrm{~m}$, de $30 \%$ de SE-30 sobre Chromosorb W 60/80 mesh. temperatura programada de 120 a $220^{\circ} \mathrm{C}$. Temperatura do detector de ionização de chama $290^{\circ} \mathrm{C}$. Nitrogênio foi usado como gás de arraste.

Identificação dos constituintes: A identificação dos constituintes foi feita por :

1 - Tempo de retenção relativa em Cromatógrafo Varian modelo $2440 \mathrm{~S}$, operando com colunas de $3 \%$ de SE-30 e $5 \%$ de Carbowax $20 \mathrm{M}$, sobre Chromosorb W, colunas de $1 / 8 \% \mathrm{x}$ $4 \mathrm{~m}$, em temperaturas variáveis, conforme o componente a identificar, utilizando-se nitrogênio como gás de arraste;
2-Ressonância magnética protônica, utilizando-se instrumento Varian modelo XL-100-12, $100 \mathrm{MHz}$;

3 - Espectrometria de Massa utilizando-se um instrumento Varian modelo $\mathrm{CH}$ 5-DF, acoplado a sistema de processamento de dados Varian SS-100 MS.

\section{AgRADECIMENTOS}

Os autores são gratos ao Dr. João Murça Pires e ao Dr. William A. Rodrigues pela coleta e identificação do material vegetal. A classificação das piperáceas foi efetuada pela Dra. Carmem Lúcia Falcão Ichaso.

\section{SUMMARY}

The major constituents of several amazonian species belongging to the families Annonaceae, Bignoniaceae, Burseraceae, Compositae, Euphorbiaceae, Labiatae, Lauraceae, Leguminosae-Papilionoideae. Myrtaceae, Piperaceae and Zingiberaceae are described.

\section{REFERÊNCIAS BIBLIOGRÁFICAS}

AlenCAR, R.; LIMA, R.A. DE; CORRÊA, R.G.C.; GOTTLIEB, O.R.; Marx, M.C.; SIlva, M.L. da; MaIA, J.G.S.; MAGalhães, M.T.

1971 - Óleos essenciais de plantas brasileiras. Acta Amazonica, 1(3) : 41-43. 
Alvarenga, M.A. de; Brocksom, U.; Castro, C.O.; Gottlieb, O.R.; Magalhães, M.T.

1977 - Neolignans from Aniba burchellii. Phytochemistry, $16: 1797-1799$.

A.RAúJo, V.C. DE; CorrêA, R.G.C.; Maia, J.G.S.; Silva, M.L. DA; GotTlieb, O.R.; MarX, M.C.; Magalhães, M.T.

1971 - Oleos essenciais da Amazônia contendo linalol. Acta Amazônica, 1(3) : 45-47.

Braz Filho, R.; Figlioulo, R.; Gottlieb, O.R.

1980 - Neolignans from a Nectandra species. Phy. tochemistry, 19(4) : 659-62.

CorrêA, R.G.; SILVA, M.L. DA; MAIA, J.G.S.; GotTlieb, O.R.; Mourão, J.C.; Marx, M.C.; Moraes, A.A. De; Koketsu, M.; Moura, L.L.; Magalhães, M.T.

1972 - Óleus essenciais de espécies do gênero Calyptranhes. Acta Amazonica, 2(3) : 53-54.

Diaz, A.M.P. \& GotTlieb, O.R.

1979 - Propiophenones from Piper marginatum. Planta Médica, 35 : 190-191.

GoTTLIEB, O.R.

1972 - Chemosystematics of the Lauraceae. Phytochemistry, $11: 1537-1570$.

Gottlieb, O.R. \& Magalhães, M.T.

1960 - Modified distillation trap. Chemist-Analyst, $49: 114$.
Moraes, A.A. de; Mourão, J.C.; Gottlleb, C.R.; Silva, M.L. DA; MarX, M. C.; Maia, J. G.S.; Magalhães, M.T.

1972b-Óleos essenciais da Amazônia contendo timol. Acta Amazonica, 2(1) : 45-46.

Moraes, A.A. DE; Rezende, C.M.A. DA M.; Bülow, M.V. Von.; Mourão, J.C.; Gottlieb, O.R.; MarX, M.C.; Rocha, A.I. da; Magalhães, M.T.

1972a - Óleos essenciais de espécies do gênero Aniba. Acta Amazonica, 2(1) : 41-44.

Mors, W.B.; Cascon, S.C.; Tursch, B.; Aplin; DURHAM

1965 - Ichthyothereol and its acetate, the active polyacetylene constituents of Ichthyothere terminalis (Spreng.) Malme, a fish poison from the lower Amazon. J. Am. Chem Soc., 87 : 5237-5241.

Naves, Y.R.; Gottlieb, O.R.; Magalhães, M.T. 1961 - Sur l'huile essentielle d'Ocotea teleiandra. Helv. Chim. Acta, 44 : 1121-1123.

Silva, M.L. da; MaIA, J.G.S.; Mourão, J.C.; Pedreira, G.; MarX, M.C.; Gottlieb, O.R.; Magallães, M.T. 1973 - Óleos essenciais da Amazônia. VI. Acta Amazonica, 3(3) : 41-42.

(Aceito para publicação em 18/09/80) 